ARTíCULO DE REVISIÓN

\title{
Modo radiólogo: una actitud
}

\section{Radiologist mode: An attitude}

\section{Gaspar A. Motta-Ramírez ${ }^{1 *}$ y Abraham Gaytán-Ortiz ${ }^{2}$}

${ }^{1}$ Servicio de Sanidad, XII Región Militar, Irapuato, Guanajuato; ${ }^{2}$ Departamento de Imagenología, Hospital Ángeles del Carmen, Guadalajara, Jalisco. México

\section{RESUMEN}

Derivado de una constante práctica médica, adoptamos como un estilo de vida, en conjunción con la pasión por el fútbol americano, con mis colegas, y en mi época de residente de la especialidad, alguna vez compartí el «beast mode», el «modo bestia», cuando me motivaron con una serie de premisas sobre nuestro quehacer como residentes, y extensivas en un futuro a nuestra práctica profesional. Mezclamos la expresión «turbo» que usábamos para motivarnos y dar celeridad a nuestro trabajo con ese «modo bestia» y creamos el «rady mode», «MODO RADIÓLOGO», creando nuestras propias reglas, definidas como UNA ACTITUD.

Palabras clave: Médico radiólogo. Educación médica. Habilidades. Ética

\section{ABSTRACT}

Derived from a constant medical practice, we adopt as a lifestyle, in conjunction with the passion for American Football with my colleagues, and in my time as a resident of the specialty I once shared the «beast mode», when they motivated me with a series of premises about our work as residents and extensive in our future to our professional practice. We mix the expression «turbo» that we used to motivate and speed up our work with that beast mode and create the «rady mode», "RADIOLOGICAL MODE», creating our own rules defined as AN ATTITUDE.

Key words: Radiologist. Medical education. Skills. Ethics. 
I keep six honest serving men (They taught me all I knew);

Their names are What and Why and When; And How and Where and Who.

Mantengo seis hombres honestos (me enseñaron todo lo que sabía);

Sus nombres son qué, por qué y cuándo; y cómo, dónde y quién.

\section{Rudyard Kipling}

Han pasado ya algunos años desde que tomé la decisión de realizar la especialidad en radiodiagnóstico ${ }^{1}$, y por ello me integré al Departamento de Radiación Ionizante del Hospital Central Militar.

En mi opinión y por mis vivencias, descubrí un lugar extraordinario donde hay una combinación fabulosa de liderazgo, disciplina, mística, profesionalismo y ética, por mencionar algunos de los valores y virtudes que todo médico, en nuestro caso, médicos militares, adscritos, y por supuesto todos los médicos residentes, aplicamos a diario, favorecido por el acceso a la más alta tecnología con la que cuenta el Departamento de Radiación Ionizante del Hospital Central Militar. Tal hecho coloca al Departamento como un centro generador de conocimiento ${ }^{2-4}$, de diagnóstico y tratamiento, más que importante dentro del grupo multidisciplinario que, diariamente, las 24 horas del día y los 365 días del año, se esfuerza por ofrecer los más altos estándares de atención médica con calidad y calidez a todos los integrantes de las Fuerzas Armadas, NUESTRA FAMILIA POR ELECCIÓN, quienes cumplen las misiones que nos exige el pueblo de México, a lo largo de nuestro territorio nacional. Este equipo de trabajo al que me refiero se motiva no solo por el concepto de servir a su nación, sino también en el personal, a lo que más amamos en nuestra vida y que es el motor que nos impulsa a todos a ser mejores cada día: NUESTRAS FAMILIAS. Así, somos afortunados al contar con dos familias, la propia y la que elegimos por elección.

Tal como versa el himno de nuestra alma mater: «EN CALLADA LABOR COTIDIANA», todos los días, el personal de profesionales de salud que labora en este departamento, que incluye desde las áreas de recepción, el personal técnico en sus diferentes áreas con sus equipos y estaciones de trabajo, culminando en las áreas de interpretación, donde siempre encontraremos a sus integrantes, el equipo de salud, en acción. En las áreas de interpretación ${ }^{5}$, santuarios de conocimiento, al cruzar su puerta, encontraremos un cambio notable y peculiar de iluminación que nos lleva a una iluminación de color azul, de ahí «cuarto azul», semiobscuro, en silencio, o quizá con un fondo de música relajante, tenue, casi imperceptible con los monitores encendidos mostrándonos imágenes médicas, invitándonos a un interesante y excitante viaje a través de una de las máquinas más complejas del universo: el cuerpo humano. Y somos nosotros, los médicos radiólogos, los que hemos aceptado el reto de conocer a la perfección la anatomía y sus variantes normales, de interpretar las imágenes para identificar aquellas enfermedades que aquejan a los seres humanos, y con una extrema meticulosidad, mediante acciones que parecen pasivas -sentados y concentrados frente a un monitor- al momento de analizar todas y cada una de las imágenes obtenidas a través de las múltiples modalidades de estudios radiológicos y de imagen, alcanzamos una gran 
actividad, dinámica, compleja al reconocer y establecer cabalmente un diagnóstico por imagen, sustentado no solo con la imagen, siendo necesario ir más allá, al recopilar y procesar la información clínica, extremadamente valiosa pero desafortunadamente escasa y otras veces nula, que nos envían nuestros colegas médicos tratantes (o clínicos o ¿cómo nombrarles?). Por ello, en múltiples ocasiones, igual o mejor aún, somos nosotros los que recabamos esa información. ¿Cuándo?, quizá con una plantilla antes de que el paciente ingrese a la sala de exploración, o tal vez al momento de realizar el estudio, pero lo esencial es NO DEJAR DE HACERLO, porque de eso depende el mayor beneficio para nuestros pacientes, que nuestros diagnósticos sean certeros, que la toma de decisiones y acciones de manejo orientadas para recuperar la salud del paciente que nos ocupa sean las mejores, y así, integrados al equipo multidisciplinario médico, materializar el objetivo real de nuestra actividad y que al hacerlo lo hagamos en un total apego a un lenguaje de respeto y bien dirigido ${ }^{6,7}$.

La tarea no es para nada fácil, mas sí por demás gratificante, y apasionante, porque no bastan uno, dos, tres, mil pacientes. Estos profesionales de la salud son incansables, inquietos y perseverantes, siempre en busca de la solución de nuevos casos, y todos son un reto, ya que todos quieren servir, entregarse en cuerpo y alma a esta noble y exigente profesión para beneficiar a otros, así que están siempre en busca de más problemas clínicos que resolver, que prever, y su ímpetu no termina ahí, siempre van más allá.

El médico radiólogo se sumerge en actividades que elevan su adrenalina con el ajetreo que se puede vivir en las salas de urgencias, mientras evaluamos un estudio de tomografía computarizada (TC) multifásico en un paciente politraumatizado en el que debemos de contribuir a la toma de decisión que se tomará en caso de encontrar lesiones: ¿quirúrgico o conservador?; o en la sala de radiología invasiva, donde se realizan infinidad de procedimientos diagnósticos y terapéuticos.

La actividad se extiende a las incontables horas de enseñanza a los médicos residentes en formación, a todas las horas de investigación y de intercambio con médicos de otras especialidades, así como con nuestra otra faceta, las comisiones que exige el deber militar.

Derivado de esta constante práctica médica, que adoptamos como un estilo de vida, en conjunción con la pasión por el fútbol americano, con mis colegas, y que, en mi época de residente de la especialidad, alguna vez compartí el «beast mode», el modo bestia, cuando me motivaron con una serie de premisas sobre nuestro quehacer como residentes y extensivas en un futuro a nuestra práctica profesional. Mezclamos la expresión «turbo» que usábamos para motivarnos y dar celeridad a nuestro trabajo con ese modo bestia y creamos el «rady mode», «MODO RADIÓLOGO», creando nuestras propias reglas definidas como UNA ACTITUD:

1. Los pacientes siempre son primero. Los pacientes acuden a cualquier centro de atención médica, incluyendo al hospital, por ayuda, por algún problema de salud. Nuestra prioridad es otorgarles atención médica lo más pronto posible y es indispensable mostrar dedicación y servicio a los intereses del paciente.

2. La familia de los colegas es como si fuera la tuya misma. 
3. La lealtad es contigo mismo y con todos los demás.

4. Sé agradecido con el que te enseña. Enseña a los que te anteceden y te rodean. No hay mejor semilla sembrada que de nuevos y mejores frutos que el conocimiento: aprende y enseña por el ejemplo ${ }^{8}$. Las entregas de guardia 9 (morning report), de un valor invaluable e intangible en esos momentos, y con un impacto sólido en nuestra formación, y que en aquellos días estaban a cargo del Dr. Reginaldo Alcántara Pedraza, quien las conducía con su estilo particular, mas con un juicio clínico-radiológico insuperable. El Dr. Reginaldo Alcántara Pedraza cedió la estafeta a otros adscritos del Departamento de Radiación Ionizante del Hospital Central Militar, como el autor del presente manuscrito, y esa actividad perdura hasta hoy.

En una editorial de la revista Archives of Pediatrics $\mathcal{E}$ Adolescent Medicine, sus autores, Ballantine y Feudtner ${ }^{10}$, sugieren 10 puntos a considerar al momento de impartir o desarrollar cualquier estrategia en educación médica. Describen cómo se han ido sustituyendo o transformando las clases tradicionales por diseños instructivos, en los que se identifican oportunidades de aprendizaje y se incorpora y se exhorta al alumno a tener un papel activo en su aprendizaje, y recalcan la importancia de no considerar estos puntos como la única estrategia de enseñanza. Su lista de cotejo es una buena guía, integrada por 10 «R», de las iniciales en inglés, para todos nuestros encuentros con estudiantes y/o residentes, el pase de visita ${ }^{10,11}$, que incluye la visita al pie de la cama del paciente $^{12}$, la entrega de guardia ${ }^{9}$, las clases o las sesiones bibliográficas. Las primeras
3 «R» se basan en un aspecto básico del aprendizaje en adultos, la reciprocidad. Las siguientes 4 se enfocan en aspectos particulares de la educación médica, y las últimas 3 al currículum médico. Las 10 «R $»^{10}$ son:

- Respeto (del inglés respectful). Se reconocen y se aprovechan las habilidades y experiencias de los alumnos.

- Responsabilidad (del inglés, responsibility). Los alumnos son autodidactas y participan en definir objetivos $\mathrm{y}$ métodos de aprendizaje.

- Relevante (del inglés, relevant). Los alumnos pueden ver si es, y por qué lo es, importante el contenido para su trabajo diario.

- Basado en investigación (del inglés, researched). El contenido está basado en la mejor evidencia disponible.

- Realista (del inglés, realistic). La integración del contenido se facilita con un ambiente de aprendizaje que simula la vida real.

- Social (del inglés, relational). El aprendizaje se sitúa en un contexto social que refleja las relaciones interpersonales presentes.

- Reflexión (del inglés, reflective). Se exhorta y se enseña a los alumnos a reflexionar sobre los eventos.

- Completo (del inglés, robust). El currículum es minucioso y completo.

- Repetición (del inglés, repetition). Los alumnos vuelven a abordar temas y habilidades desde distintas perspectivas.

- Riguroso (del inglés, rigorous). Evaluación rigurosa de alumnos y currículum para asegurar calidad.

5. La decisión de realizar la especialidad en radiodiagnóstico y de integrarme a un 
programa de residencia me replanteó innumerables aspectos de mi vida personal, sobre todo reevaluar el cómo aprendí y cómo debería enfrentar el nuevo reto $^{13}$. Reconsideré el hábito de sentirme ofendido $^{14}$, debía comprender las nuevas expectativas a las que debía someterme y crecer en ellas, sobre todo ante el análisis de incidentes críticos para aprender de mis errores ${ }^{15-18}$.

6. Siempre entablar la relación médicopaciente. Derivada de ella, recabar, siempre que la condición de nuestros pacientes lo permita, la información clínica extensa y detallada, que complementará seguramente nuestros diagnósticos por imagen, y si la condición del paciente no lo permite, agotar todos los medios posibles para obtenerla, si es necesario, tomar un teléfono y contactar al médico tratante, o mejor aún, acudir a entrevistarnos en persona con él. No titubear en asegurar que ese esfuerzo resultará en un mejor diagnóstico y pronóstico para nuestros pacientes.

7. La realimentación debe ser oportuna ${ }^{19}$. El conocimiento NO ES FINITO. Siempre hay que estar investigando y aprendiendo, así como ratificar lo ya aprendido. El nivel de conocimientos, teórico y práctico, adquirido en el Departamento de Radiación Ionizante del Hospital Central Militar es excelente, logrado a través un ritmo óptimo, individualizado y con calidad en el aprendizaje.

8. El «MODO RADIÓLOGO» NO TERMINA. La interpretación de los estudios radiológicos y de imagen no es intuitiva. Es necesario saber lo que se busca; saber dónde buscar y qué es lo que se busca9. Y luego de encontrar un hallazgo, busco otros similares. Los 10 Mandamientos para evitar errores en la interpretación de estudio radiológico ${ }^{20}$ :

- Dos vistas: Una vista siempre es muy poco, deben de solicitarse dos incidencias.

- Dos anormalidades: Si ve una anormalidad busque siempre la segunda ${ }^{21}$.

- Dos articulaciones: Solicite siempre la articulación superior a la que se estudia.

- Dos lados: Si no está seguro o está interpretando una radiografía difícil, siempre compare.

- Dos vistas diferentes son mejor: La TC ha reemplazado a la radiología en muchas situaciones convencionales.

- Dos tiempos: Si están a su disponibilidad, siempre compare con estudios previos.

- Dos visitas: Reprograme otra visita para valorar nuevamente al paciente.

- Dos opiniones y dos registros: Si tiene duda, siempre valore el estudio con un colega.

- Dos especialistas: Si tiene duda, siempre busque al especialista afín a la patología que está valorando, con el estudio que se trate.

- Dos investigaciones: Siempre considere realizar ultrasonido, TC o resonancia magnética.

9. Reforzar el concepto de profesionalismo, entendido como el conjunto de conocimientos, habilidades, principios y valores que sustentan una práctica idónea de la medicina en el marco de los más elevados estándares de calidad científica, ética y humanista ${ }^{22,23}$. 
10. Reconocer que es el momento para crear $y$ favorecer el enriquecimiento de las competencias profesionales: clínicas o del cuidado del paciente, conocimiento médico, de comunicación, éticas/ profesionalismo, docentes, de investigación y administrativas 22,23 .

11. Responsabilidad diagnóstica, calibrando los recursos que se tienen al alcance de la mano. Tener conciencia de la repercusión de sus decisiones en relación con la distribución y el uso de los recursos. Utiliza con racionalidad los recursos; piensa siempre en términos de eficiencia: elige la exploración o tratamiento decisivo entre los menos arriesgados y menos caros. Valora con otros profesionales el significado de ciertos resultados o la evolución de los enfermos.

12. El medico radiólogo es el consultor dinámico del hospital ${ }^{24}$. El trabajo en equipo $y$ de forma multi e interdisciplinaria siempre es mejor. Por ello es imprescindible participar en comités multidisciplinarios, acudir a las sesiones de los servicios, a las sesiones generales del hospital y a congresos de otras especialidades, y ello implica ${ }^{11}$ :

- Capacidad y voluntad para liderear la gestión clínica.

- Tener una buena capacidad de trabajo y una actitud positiva en el seno de equipos interdisciplinarios y multiprofesionales.

- Seguir los principios básicos ${ }^{25}$ para llevarse bien con su clínico, que además de los 9 que se señalan, debe incluirse el que se realice un estudio descriptivo de los hallazgos en las radiografías, que informemos de forma clara y comprensible sobre los hallazgos de las imágenes obtenidas en los diferentes estudios de imagen y que seamos un profesional interesado, colaborador y conocedor de nuestras responsabilidades y limitaciones.

- Adquirirelcompromiso deexcelencia con el aprendizaje permanente.

- Las notas de los estudios deben contener: fecha y hora del estudio, identificación del solicitante, estudio solicitado, problema clínico en estudio, resultados, incidentes, identificación del personal que realiza el estudio, nombre completo y firma del personal que informa.

13. En el enfoque diagnóstico de los problemas, siempre elegir la hipótesis más razonable; aunque también es pertinente considerar hipótesis sofisticadas, pero lógicas.

14. El «MODO RADIÓLOGO» toma decisiones con rapidez, conoce sus límites $\mathrm{y}$ evita decisiones que sabe que le sobrepasan; en ocasiones, decisiones intuitivas pero oportunas.

15. No solo tiene una dedicación excepcional, sino que profundiza en el conocimiento de la patología de los pacientes y en otras actividades del servicio. Propone al resto del equipo y dinamiza la puesta en marcha de actividades asistenciales, docentes y de investigación.

16. Tiene una dedicación horaria por encima de su jornada laboral. Alto nivel de responsabilidad. El médico radiólogo conecta perfectamente con las familias y los pacientes, es tolerante, toma interés por sus problemas personales y sociales, y dedica tiempo extra a discutir aspectos médicos y otras preocupaciones en el entorno familia-paciente. 
17. Relaciones amigables con el equipo de trabajo; toma interés y participa activamente en los problemas, actividades, toma de decisiones e iniciativas que implican al equipo; está totalmente integrado ${ }^{25}$.

18. El «MODO RADIÓLOGO» se nutre. En la publicación de Deitte $^{26}$ se señala y se enriquece el enfoque de capacitación y supervisión a los médicos residentes de la especialidad, futuros médicos radiólogos, para tener herramientas para evaluar su progreso. Además, es importante considerar y sumar las destrezas que dan valor a la práctica cotidiana ${ }^{27}$, así:

- Colaborar como miembro de un equipo interprofesional ${ }^{26}$, construir una buena relación con otras especialidades ${ }^{27}$.

- Realizar exámenes de triajes y establecer protocolos.

- Interpretar los exámenes y priorizar un diagnóstico diferencial.

- Comunicar efectivamente ${ }^{27}$ los resultados de los exámenes.

- Recomendar los pasos apropiados para integrar calidad, elaborar informes significativos, y comunicar con certeza e incertidumbre.

- Obtenerel consentimiento informado, mantener la seguridad radiológica, evitar repetir exámenes innecesarios ${ }^{27}$ y realizar los procedimientos.

- Manejar a los pacientes después de las imágenes y los procedimientos.

- Formular preguntas clínicas y recuperar evidencia para atención anticipada al paciente

- Comportarse profesionalmente y reducir los retrasos en la atención al paciente $^{27}$.
- Facilitar el flujo de pacientes ${ }^{27}$, informar sobre el costo de la imagen ${ }^{27}$, e identificar fallas del sistema y contribuir a una cultura de seguridad y mejora ${ }^{26}$.

19. $\mathrm{El}$ «MODO RADIÓLOGO» no se detiene: Solo puedes ser bueno en algo si practicas mucho; tienes que mostrar lo que sabes. ¡Hay tantas cosas que aprender! y quienes te rodean no quieren saber lo inteligente que eres, quieren saber lo inteligentes que $\mathrm{SON}$.

20. El «MODO RADIÓLOGO» se actualiza y se moderniza: Por ejemplo, el Dr. Safwan Halabi del Henry Ford Health System, da 10 consejos para una atención centrada en el paciente, y las estrategias de las redes sociales ocupan un lugar destacado en esos 10 consejos para los médicos radiólogos que buscan otorgar una atención centrada en el paciente ${ }^{28}$.

- Aproveche el registro de salud personal, ya que permite que los médicos radiólogos se conecten con los pacientes mediante el registro electrónico de salud, y es factible el utilizarlo para publicar informes de radiología, laboratorios y notas para los pacientes. Los pacientes también pueden programar exámenes de radiología y comunicarse con sus médicos, incluido su médico radiólogo.

- Entregue su tarjeta de presentación. El Dr. Safwan Halabi sugiere tratar de conocer al menos a un paciente y/o colega cada día. Esta acción debe incluir el dar su número de teléfono directo, dirección de correo electrónico y perfiles, por ejemplo, de LinkedIn. 
- Proporcione un número de teléfono directo y un correo electrónico en su informe de radiología. Esto permitirá a los pacientes contactar a los médicos radiólogos directamente si tienen alguna pregunta sobre el informe y así brindar a los médicos remitentes una opinión basada en evidencia ${ }^{27}$.

- Compartir imágenes. El Dr. Safwan Halabi se refirió al proyecto Image Share de la Radiological Society of North America (RSNA) como un ejemplo de intercambio de imágenes en acción. Los pacientes también pueden solicitar la posibilidad de subir imágenes a las redes sociales. Señaló que una compañía, Paxeramed, ha comenzado a incluir enlaces de redes sociales en su software PACS para permitir la carga de imágenes clave en Facebook o Box. com

- En lo que se refiere a su red social, aconseja el Dr. Safwan Halabi mantener el contenido fresco y diverso. Use todos los medios de comunicación social y permita que los invitados dejen comentarios sobre su visita, buena o mala. Debido a que cada canal de redes sociales parece tener su propio tipo de usuario, es una buena idea estar en todas las plataformas principales, incluidas YouTube, Facebook, Twitter, LinkedIn, Google Plus, Pinterest e Instagram.

- Use fotos y vídeos. Esto podría incluir crear contenido o compartir vídeos de RadiologyInfo.org para proporcionar orientación e instrucciones a los pacientes antes de someterse a procedimientos de radiología.

- Interactúe $\operatorname{con}^{27}$ y obtenga la opinión del paciente. Incluya a los pacientes en la toma de decisiones.

- Obtenga aportes de colegas para desarrollar y seleccionar contenido.

- Sea transparente. Es importante compartir éxitos y fracasos; las redes sociales pueden ayudar a proporcionar transparencia a los pacientes. Nunca es fácil de hacer, más la disculpa es personal y debe hacerse pública cuando se cometen errores. Las disculpas que aceptan la responsabilidad son más efectivas que simplemente expresar simpatía. Sin embargo, las expresiones sinceras de simpatía pueden tener muchos de los efectos positivos de las disculpas que aceptan la responsabilidad, aunque no en el mismo grado.

- Evolucionar. El Dr. Safwan Halabi aconseja a los médicos radiólogos que se manten1gan al día con los formatos de redes sociales más recientes y que estén listos para comunicarse con los pacientes y aceptar la comunicación de ellos. También recomienda proporcionar a los pacientes informes radiológicos ricos en imágenes y en datos, y permitirles descargar, almacenar y compartir sus imágenes en la nube ${ }^{28}$.

21. Por último y sin dejar de ser importante: Seamos visibles ${ }^{29}$. Presentémonos con el paciente, que el paciente sepa que lo está atendiendo un «médico radiólogo», «médico imagenólogo» $\mathrm{O}$ «médico residente radiólogo o imagenólogo", y cuando finalicemos el estudio, aclaremos sus dudas y, si lo cuestiona, 
comuniquémosle nuestro hallazgos, de una manera breve y concisa, en lenguaje común, que NO se retire con incertidumbre del departamento de radiología, que obtenga su diagnóstico o por lo menos una aproximación. Para el paciente solo existe quien da la cara ante él, todos los demás son «entes borrosos» que recuerda someramente dentro de su atención médica.

Es evidente que el mundo ha cambiado y continúa cambiando constantemente. Y ello afecta tanto a las relaciones personales como a las laborales y, por consecuencia, serán necesarias distintas habilidades sociales y laborales a las de hoy en día. El ambiente laboral se verá afectado por las nuevas tecnologías y otros factores, tales como los cambios tecnológicos y demográficos. Por ello es indispensable transmitir a nuestro residentes, futuros adscritos del siglo $\mathrm{XXI}^{30}$ que:

- El teletrabajo y el trabajo serán flexibles. Las personas trabajarán desde distintos sitios y con horarios más flexibles.

- Big Data y las nuevas tecnologías. Estas afectarán el funcionamiento, así como la manera de aproximarse a los cambios en el mercado.

- La inteligencia artificial. Este ha sido uno de los temas más discutidos y tendrá aún más influencia en el ambiente de trabajo para 2020.

- Habilidades laborales necesarias. Todos los factores mencionados anteriormente harán el que sea necesario adaptarse a los cambios y a desempeñarse de manera distinta. Las habilidades tendrán que responder a tecnologías y a una distribución distinta del trabajo. Esto se evidencia con el establecimiento del internet como norma.

- Pensamiento crítico. Descartes dijo «Pienso, luego existo». Piense críticamente, y usted será todo lo que puede ser.

- Creatividad.

- Gestión de equipo e inteligencia emocional. Es claro que estas dos habilidades van de la mano. Es necesario utilizar la inteligencia emocional y técnicas como el mindfulness para gestionar mejoras en el equipo de trabajo y de sus relaciones laborales.

- Toma de decisiones. Esta habilidad es clave en cualquier ambiente de trabajo. En la época actual, con tantas distracciones presentes en el día a día, el médico radiólogo debe tomar decisiones sabias con respecto a su tiempo.

- Negociación. Esta es una habilidad esencial.

Así, finalmente como lo señala la publicación «Ética médica e imagenología» 31 , la ruta de acción en la que podemos cumplir los preceptos éticos de nuestra especialidad y perpetuar el «MODO RADIÓLOGO» (rady mode) es promover la comunicación entre todos los implicados (siempre amplia, crítica, propositiva y multidireccional), así como incidir desde la educación médica inicial y dentro de nuestra y otras especialidades, lo que nos permitirá ser parte de una generación diferente de médicos, una generación mejor aún, una generación que incluya en su currículum, como lo escribe Xavier Marcet en su artículo de La Vanguardia «Habilidades 2030»32, cinco habilidades básicas que son: pensar, vender, dar operatividad a los relatos, aprender 
y respetarse. En un mundo cambiante y complejo necesitamos líderes (recordemos que todos en determinado lugar y momento lo seremos) que piensen y que duden (no nos fiemos de aquellos que ante tanta complejidad no dudan). Más necesitamos que además escriban y decidan. De la gente que no escribe no podemos saber si piensa. Y me refiero a escribir, no a mandar tweets o whatsapps. Pensar es la base de la estrategia y la innovación. Pensar como hábito. Papel en blanco. Esquemas que fijen la fugacidad de las ideas. Pros y contras.

Consideramos que nuestra especialidad «vende mucho», pero tenemos que estar, interactuar, participar, opinar, como ya lo manifestamos antes, «seamos visibles» ${ }^{29}$. Las aplicaciones inteligentes cambiarán los contextos de las relaciones humanas, afinarán los esfuerzos comerciales, pero la habilidad de lograr comunicarse es perpetua. Los médicos radiólogos debemos mejorar nuestras relaciones con todos los integrantes del equipo de salud, no solo con los médicos tratantes. Debemos ser visibles, activos, participativos, colaboradores y con actitud positiva para con todos los miembros integrantes de nuestras áreas de trabajo. Así, lograremos asegurar nuestra visibilidad, como también la necesidad de nuestras acciones, y para ello debemos hacer más accesibles a los médicos clínicos y a los pacientes el cómo realizamos nuestro trabajo y cómo es que ese producto es una parte fundamental en la toma de decisiones clínicas hoy por hoy en el mundo. El cómo enfoquemos esta energía para desarrollar e implementar esta transición hacia el frente del trabajo clínico nos definirá en el futuro ${ }^{29}$.

En lo que se refiere a dar operatividad al relato, es la habilidad de los «doers», de los hacedores, personas que alcanzan la excelencia en un campo determinado por la práctica reiterada y de manera continua en un campo, como lo describe en el libro «Los fuera de serie» de Malcolm Gladwell $^{33}$. Sin ellos todo sería aspiracional. Los discursos acompañan, pero los hechos, las operaciones, los productos, los servicios, son la narrativa definitiva. Es decir, la interpretación de un estudio por el responsable del mismo, el «doer» es el médico radiólogo.

El aprender y el desaprender son la base de la adaptación y el motor de crecimiento de las personas y las corporaciones. Aprender tiene que ver con transformación. Alvin Tofler, reconocido por sus teorías sobre tecnologías, la revolución digital y su impacto en ciudadanos y culturas de todo el mundo, señala que no hay más opción que adaptarse y nunca dejar de actualizarse ya que «Los analfabetos del siglo XXI no serán aquellos que no sepan leer ni escribir, sino aquellos que no puedan aprender, desaprender y reaprender» ${ }^{34}$.

Y finalmente, crea respeto. La capacidad de tejer relaciones positivas. De crear conexiones donde el propósito empieza con el respeto a los demás, ya sea paciente, médico tratante, médicos residentes, otros médicos radiólogos. Crear relaciones de respeto es la base de cualquier acción humana y crea un crecimiento sostenible. El respeto es lo que permite crear el sentido de pertenencia. El respeto es lo que queda cuando te vas. Cuando nos vayamos solo quedará el «MODO RADIÓLOGO».

\section{AGRADECIMIENTOS}

Los autores agradecen a la Escuela Militar de Graduados de Sanidad y al Departamento de 
Radiación ionizante del Hospital Central Militar.

\section{CONFLICTO DE INTERESES}

Los autores declaran no tener ningún conflicto de intereses.

\section{FINANCIAMIENTO}

Los autores declaran que no recibieron patrocinio para llevar a cabo este artículo.

\section{RESPONSABILIDADES ÉTICAS}

Protección de personas y animales. Los autores declaran que para esta investigación no se han realizado experimentos en seres humanos ni en animales.

Confidencialidad de los datos. Los autores declaran que en este artículo no aparecen datos de pacientes.

\section{Derecho a la privacidad y consentimiento} informado. Los autores declaran que en este artículo no aparecen datos de pacientes.

\section{BIBLIOGRAFÍA}

1. Motta-Ramírez GA. Ser médico radiólogo. Slideshare (Internet). Último acceso: 10 de Octubre 2020. Disponible en: https://es.slideshare.net/ betomotta/17-ix-2013-ser-mdico-radiologo

2. Dorio P. Why radiology is the cornerstone of any hospital. KevinMD.com (Internet). Último acceso: 10 de Octubre 2020. Disponible en: https:/ /www. kevinmd.com/blog/2011/10/radiology-cornerstone-hospital.html

3. Dorio P. Why radiology should become more like McDonal's. KevinMD. com (Internet). Último acceso: 10 de Octubre 2020. Disponible en: https:/ / www.kevinmd.com/blog/2010/08/radiology-mcdonalds.html

4. Motta-Ramírez GA. La educación actual del residente en la especialidad de Radiología e Imagen.Slideshare (Internet). Último acceso: 10 de Octubre 2020. Disponible en: https://es.slideshare.net/betomotta/la-educacionactual-del-residente-en-la-especialidad-de-rx-e-i-gamr
5. Motta-Ramírez GA. Porque los médicos radiólogos interpretan con luz baja. Slideshare (Internet). Último acceso: 10 de Octubre 2020. Disponible en: https:/ / es.slideshare.net/betomotta/porque-los-mdicos-radiologos-interpretan-con-luz-baja

6. Levine MS. Laufer's rules. Applied Radiology (Internet). Último acceso: 10 de Octubre 2020. Disponible en: https://appliedradiology.com/articles/ laufers-rules

7. Galasinski D. Language matters: a linguist's view on medicine. Sex Transm Infect. 2017;93(7):456-457.

8. LaCombe MA. Teaching and learning by example. Ann Intern Med. 2018;168(7):521-522.

9. Schiffman FJ. Morning report and work rounds: Opportunities for teaching and learning. Transactions of the Amer Clin and Climatological Assoc. 1995;107:275-286.

10. Ballantine A, Feudtner C.The 10 R's of clinician education. A checklist. Arch Pediatr Adolesc Med. 2010;164(4):389-390.

11. Abdool MA, Bradley D. Twelve tips to improve medical teaching rounds. Medical Teacher. 2013;35:(11):895-899.

12. Ramani S. Twelve tips to improve bedside teaching. Medical Teacher. 2003;25(2):112-115.

13. Motta-Ramirez GA, Castillo-Lima JA, Gómez del Campo. Radiólogos, clínicos y atención médica. Rev Sanid Militar. 1997;51:247-250.

14. Engelke M. El hábito de sentirnos ofendidos. Dynamic power coach. (Internet). Último acceso: 10 de Octubre 2020. Disponible en: https://www. dpcoach.com.mx/habito-sentirnos-ofendidos/

15. Renfrew DL, Franken EA Jr, Berbaum KS, Weigelt FH, Abu-Yousef MM. Error in radiology: classification and lessons in 182 cases presented at a problem case conference. Radiology. 1992;183(1):145-50.

16. Almendro-Padilla C, Costa-Alcaraz AM. Análisis de incidentes críticos: una herramienta para aprender de los errores. Educ Med. 2018;19(1):60-63.

17. Portela-Romero M, Bugarín-González R, Rodríguez-Calvo MS. Error humano, seguridad del paciente y formación en medicina. Educ Med. 2019;20(S1):169-174.

18. Bruno AM, Walker EA, Abujudeh HH. Understanding and confronting our mistakes: The epidemiology of error in radiology and strategies for error reduction. Radiographics. 2015;15:1668-1676.

19. Motta-Ramírez GA. Realimentación. Slideshare (Internet). Último acceso: 10 de Octubre 2020. Disponible en: https://es.slideshare.net/betomotta/ realimentacion-feedback

20. Touquet R, Driscoll P, Nicholson D. Teaching in accident and emergency medicine: 10 commandments of accident and emergency radiology. BMJ. 1995;310:642-645.

21. Roger LF. Keep Looking: Satisfaction of Search. AJR. 2000;175:287.

22. Blank L. Medical professionalism in the new millennium: a physicians' charter. Medical Professionalism Project. Ann Intern Med. 2002;136:243-246

23. Taylor C, Farver C, Stoller JK. Perspective: Can emotional intelligence training serve as an alternative approach to teaching professionalism to residents? Acad Med. 2001;86:1551-1554.

24. Motta-Ramírez GA. La práctica y el ejercicio profesional del médico radiólogo en el siglo XXI. Slideshare (Internet). Último acceso: 10 de Octubre 2020. Disponible en: https://es.slideshare.net/betomotta/ la-practica-y-el-ejercicio-profesional-del-medico-radiologo-en-elsiglo-xxi

25. Motta-Ramírez GA. Principios básicos para llevarse bien con su clínico. Slideshare (Internet). Último acceso: 10 de Octubre 2020. Disponible en: https://es.slideshare.net/betomotta/como-llevarse-bien

26. Deitte LA, Gordon LL, Zimmerman RD, Stern EJ, McLoud TC, Diaz-Marchan PJ, Mullins ME. Entrustable professional activities: Ten things radiologists do. Acad Radiol. 2016;23:374-381.

27. Margolis NE, Mackey RA, Sarwar A, Fintelmann FJ.15 Practical Ways to Add Value in Daily Practice: An Imaging 3.0 Primer for Trainees. J Am Coll Radiol. 2015;12(6):638-640. 
28. Ridley EL. Top 10 social media tips for radiologists. AuntMinnie.com (Internet). Último acceso: 10 de Octubre 2020. Disponible en: https://www. auntminnie.com/index.aspx?sec=sup\&sub=imc\&pag=dis\&ItemID=107455

29. Motta-Ramirez GA. Vivir la medicina. Aprendiendo a ver, a ser visible. An Radiol Méx. 2016;15(2):85-87.

30. Institute for the future for the University of Phoenix Research Institute. Future work skills. www.iftf.org (Internet). Último acceso: 10 de Octubre 2020. Disponible en: http://www.iftf.org/uploads/media/SR-1382A_ UPRI_future_work_skills_sm.pdf

31. Lozano-Zalce H. Ética médica e imagenología. Acta Médica Grupo Ángeles. 2017;15(1):5-7.
32. Marcet X. Habilidades 2030. pressreader.com (Internet). Último acceso: 10 de Octubre 2020. Disponible en: https://www.pressreader.com/spain/lavanguardia-dinero/20200223/281509343213279

33. Gladwell M. Fuera de serie (Outliers). Academia.edu (Internet). Último acceso: 10 de Octubre 2020. Disponible en: https://www.academia.edu/34818700/Fuera_de_serie_-_Malcolm_Gladwell? auto=download

34. Inma Capo Panades. instituto-pnl.com.@inmacapo (Internet). Último acceso: 10 de Octubre 2020. Disponible en: https://www.pinterest.com.mx/ pin/570549846512179181/ 